\title{
Ethical considerations about artificial intelligence for prognostication in intensive care
}

Michael Beil ${ }^{1 *}$ D, Ingo Proft ${ }^{1,2}$, Daniel van Heerden ${ }^{3}$, Sigal Sviri ${ }^{4}$ and Peter Vernon van Heerden ${ }^{4}$

\author{
* Correspondence: beil@doctors.org. \\ uk \\ ${ }^{1}$ Institute of Health Sciences at \\ PTHV, Pallottistr. 3, 56179 Vallendar, \\ Germany \\ Full list of author information is \\ available at the end of the article
}

\begin{abstract}
Background: Prognosticating the course of diseases to inform decision-making is a key component of intensive care medicine. For several applications in medicine, new methods from the field of artificial intelligence (Al) and machine learning have already outperformed conventional prediction models. Due to their technical characteristics, these methods will present new ethical challenges to the intensivist.

Results: In addition to the standards of data stewardship in medicine, the selection of datasets and algorithms to create Al prognostication models must involve extensive scrutiny to avoid biases and, consequently, injustice against individuals or groups of patients. Assessment of these models for compliance with the ethical principles of beneficence and non-maleficence should also include quantification of predictive uncertainty. Respect for patients' autonomy during decision-making requires transparency of the data processing by Al models to explain the predictions derived from these models. Moreover, a system of continuous oversight can help to maintain public trust in this technology. Based on these considerations as well as recent guidelines, we propose a pathway to an ethical implementation of Al-based prognostication. It includes a checklist for new Al models that deals with medical and technical topics as well as patient- and system-centered issues.

Conclusion: Al models for prognostication will become valuable tools in intensive care. However, they require technical refinement and a careful implementation according to the standards of medical ethics.
\end{abstract}

Keywords: Artificial intelligence, Machine learning, Intensive care, Medical ethics, Prognostication

\section{Background}

Prognosticating the course of critical illnesses and predicting the impact of interventions are major pillars of decision-making in intensive care [1,2]. This involves models which describe the underlying disorders in reductionist ways. Simple mechanistic types of models are based on causal relationships, e.g., hypotension in a dehydrated patient will respond to fluid resuscitation. A substantial number of individuals in intensive care, however, present with more complex disorders [3]. The critical condition in these patients results from various processes evolving at different time scales and interacting in non-linear and stochastic ways. Due to the large number of parameters, predictions based on mechanistic modeling become impractical. The intensivist then has to apply

(c) The Author(s). 2019 Open Access This article is distributed under the terms of the Creative Commons Attribution 4.0 International License (http://creativecommons.org/licenses/by/4.0/), which permits unrestricted use, distribution, and reproduction in any medium, provided you give appropriate credit to the original author(s) and the source, provide a link to the Creative Commons license, and indicate if changes were made. 
statistical techniques comparing the most salient traits of an individual case with reference classes, i.e., homogeneous cohorts of patients with the same dominant disorder(s). Due to the necessary reduction and, thereby, selection of the patient's features to compare, these models match the characteristics of individual cases with only variable accuracy and prognostication becomes imprecise [4].

New prognostication techniques from the field of artificial intelligence (AI) and machine learning can process large amounts of data and have already demonstrated promising results in clinical studies [5-7]. For example, mortality prediction in cohorts of patients after cardiac arrest was substantially better with an area under the receiver operating curve (AUROC) of 0.87 in comparison to conventional prediction scores with an AUROC of 0.8 [8]. This difference was reproduced by predicting mortality in intensive care patients with the AUROC for the new techniques being 0.88 and that for conventional scores 0.78 or less [9]. Regarding these developments, it appears to be irresponsible not to consider making these new techniques part of everyday practice [10]. The efficient use and fast proliferation of AI applications in other parts of society, fed by advances of machine learning technologies and autonomous decision-making, underline the acuity of this topic [11]. In medicine, however, there are no AI systems for prognostication in routine use so far. In contrast to classifying existing data, such as retinal images [12], prognostication of future events is complicated by interferences which are still to happen. This makes predictions especially sensible to environmental conditions and adds an additional layer of uncertainty. There are also concerns about the biases and robustness of new AI techniques caused by the principal design of these technologies, i.e., their dependency on the properties of data samples used for machine learning. These issues lead to a number of ethical problems for prognostication in clinical practice. This paper will discuss these problems as well as their impact on intensive care medicine.

\section{Al and machine learning}

AI refers to computer-based techniques making decisions which require human-like reasoning about observed data [13]. Historically, AI technologies were based on explicit rules and logic trying to simulate what was perceived to be the thought processes of human experts [6]. However, many prognostic questions in medicine are "black box" problems with an unknown number of interacting processes and parameters. Applying a restricted number of rules does not match the complexity of these cases and, therefore, cannot provide a sufficiently accurate prognostication. New types of AI are based on machine learning and better suited to this task [14]. They include artificial neural networks (ANNs), random forest techniques, and support vector machines.

The main aspect of machine learning is that the specific parameters for an underlying model architecture, such as synaptic weights in ANNs, are not determined interactively by humans. Instead, they are learned using general-purpose algorithms to obtain a desired output in response to specific input data [15]. The structural characteristics of a particular method, such as the layered architecture of an ANN, together with the associated set of fitted parameters constitutes a model that can be used for making predictions for new data inputs [16]. Adapting the architecture of a particular AI technology to specific types of input data can enhance predictive performance. For example, "recurrent" ANNs, such as long short-term memory, are constructed in a way to 
improve sequential data processing to capture temporal dependencies [17]. Of note, there has not yet been an algorithm developed to determine which type and architecture of the AI model would be optimal for a specific task [18].

For each particular model architecture, there are many different and equally good ways of learning from the same data sample. The machine learning algorithms usually do not recognize a single best combination of model parameters if such an optimum exists at all [6]. They can infer one or several plausible parameter sets to explain observed data presented during the learning phase [16]. This technology, therefore, is considered data-driven. It can detect emergent patterns in datasets, but not necessarily causal links [19].

\section{Datasets in machine learning}

AI models for the purpose of prognostication are based on input of static or dynamic data, i.e., time series, or a combination thereof. Accumulation of data over time to document trends can enhance prediction accuracy [5]. Of great importance is the processing of heterogeneous data from electronic health records [20]. Due to the dependency of machine learning on the properties of datasets for training, issues of data quality and stewardship are becoming crucial. In addition to the availability and usability, reliability of data is an important topic. It encompasses integrity, accuracy, consistency, completeness, and auditability of datasets [21].

Useful output data for prognostication are either numbers representing probabilities of future events, time intervals until these events, or future trajectories of clinical or functional parameters. In addition to predictions of death vs. survival, prognosticating quality of life trajectories is now becoming more important for guiding decisionmaking, notably in the elderly [22]. Although the general concept of quality of life is difficult to operationalize, there are readily observable markers, such as the ability to perform activities of daily living [23], frailty [24], or cognitive capacity [25], which may serve as surrogates.

\section{Specific problems of machine learning}

Both the structural characteristics of the models as well as the machine learning process itself make these new AI technologies different from previous approaches to prognostication. The lack of explicit rules of how machine learning operates prevents an easy interpretation by humans. This problem is most pronounced in ANNs due to the multitude of non-linear interactions between network layers [6]. Moreover, some model types, notably ANNs, are known to produce unexpected results or errors from previously known input data with some, apparently irrelevant modifications that might be undetectable by human observers ("adversarial examples"). Whether a particular error is a one-off "bug" or evidence of a systemic failure might be impossible to decide with poorly interpretable machine learning methods [26]. This also makes generalization of AI models potentially dangerous and, therefore, is considered a major problem for many applications [27, 28].

The learning process itself can be compromised by over- and underfitting models to the specific characteristics of training data. Underfitting models already fail to account for the variability of training data. Overfitting leads to a good performance with training data, but can eventually harm the robustness of the model in future real world 
applications with some distributional shifts of input data, e.g., due to variable practice patterns in different countries [29].

\section{Ethical considerations}

There are a growing number of recommendations and guidelines dealing with the issue of ethical AI [30]. The European Commission has recently published guidelines for ethical and trustworthy AI. According to these guidelines, special attention should be focused on situations involving vulnerable people and asymmetries of information or power. In addition to adhering to laws and regulations and being technically robust, AI must be grounded in fundamental rights, societal values, and the ethical principles of explicability, prevention of harm, fairness, and human autonomy [31]. These principles echo the prima facie principles of medical ethics-beneficence, non-maleficence, justice, and human autonomy [32] - which are aimed at protecting vulnerable patients in the context of uncertainty and social hierarchies.

\section{The principles of beneficence and non-maleficence}

The concepts of human dignity and sanctity of life imply that the application of information technology in medicine must be beneficent and non-maleficent for the individual patient. However, the specific AI use case of prognostication has the potential to violate these principles. Communicating probabilities derived from cohort studies to individual patients carries the risk of false hope, false despair, or continuing uncertainty. A falsely optimistic prognosis based on, for example, an unsuitable dataset for training an AI model could trigger futile, i.e., potentially inappropriate interventions. A falsely pessimistic prognosis could become a self-fulfilling prophecy when left unchecked [33]. The problems of accuracy and uncertainty apply to all probabilistic methods for prognostication and not only to AI. A way to solve this dilemma is to personalize probabilities as much as possible, e.g., by taking into account more features describing the individual circumstances of patients. The use of new AI techniques, such as recurrent ANNs, to extract prognostic information from longitudinal datasets [34] may serve that purpose. This approach is of particular interest, since most patients in intensive care are not in a steady state. Thus, the individual time course of a condition could be more informative and its analysis more predictive and, hence, more beneficial for the individual patient, than the analysis of data from a singular point in time.

\section{The principle of justice}

The principle of justice deals with the distribution of resources within a society and non-discrimination of individuals. Non-intentional injustice to individuals has become an important issue for AI. Ranking algorithms are of particular concern in many fields of application. Due to the data-driven nature of AI techniques, the selection of datasets for training is a major source of discrimination. The following scenarios are of particular importance in this regard:

- Cultural biases can be inadvertently propagated from different communities by overlooking implicit rules ingrained in the social or professional framework of a specific environment [10]. This problem has already been recognized for 
conventional prognostication scores [35] which have then been modified to incorporate environmental characteristics [36].

- Algorithms may assign a low chance of survival to previously disadvantaged patient groups whose social status had correlated with a discriminatory biomarker, e.g., body weight. Suitable strategies for testing models and continuously auditing results will reduce that risk [31].

- The historical definition of empirical disease categories pushes the specific condition in an individual into a potentially inappropriate frame to guide further management. New research based on unsupervised learning methods has identified new and prognostically meaningful disease phenotypes that fit individual cases more accurately [37, 38]. For example, Seymour et al. [37] identified four novel phenotypes of sepsis which differ with respect to biomarkers and mortality. By using this new stratification, patients with sepsis could eventually receive treatment that is more adaptive in timing and intensity.

A possible approach to prevent already recognized biases is to exclude certain parameters, such as age or gender, from the training of AI models. Importantly, this is a conscientious decision within the society that introduces new biases and might also be associated with a price to pay, such as a substantial reduction of model performance and, therefore, its usability. Conflicts may occur between the different levels of justice (societal vs. individual) and could eventually violate the respect for patients' autonomy (see below).

\section{The principle of patients' autonomy}

The respect for patients' autonomy acknowledges the capacity of individuals for selfdetermination and the right to make choices based on his/her own values and beliefs [39]. It is regarded by many ethicists as first among equals. Not surprisingly, some authors, however, consider that statement problematic, especially in relationship to the principle of (distributive) justice [32]. The respect of patients' or their surrogates' deliberate choices also encompasses dealing with seemingly irrational decisions. The spectrum ranges from refusal of life saving treatment in a recently healthy individual to demands for interventions in a patient dying from an incurable disease. This opens the debate for an ethical analysis of personal behavior [40]. Guidelines for responding to requests for potentially inappropriate (futile) therapies in intensive care emphasize the importance of this issue [41]. It is important to note that predicting the course of a critical condition in individuals remains uncertain on principle. The fundamental problem of falsifying individual beliefs in future events cannot be solved by new AI technologies.

An autonomous decision by the patient or a surrogate decision-maker requires a sufficient understanding of the relevant medical information as well as of the decisionmaking process within the medical community, such as adherence to guidelines. The latter condition enables a dynamic dialog, i.e., shared decision-making, during the often unpredictable course of critical diseases. However, it is unrealistic to assume that these conditions can be fulfilled in every case. Hence, the trust between patients, surrogates, and physicians still is a major pillar of decision-making in intensive care. Traditionally, the burden of ethical decision-making is put onto the medical staff who must guarantee 
that the patient or his/her surrogate is able to make a decision to the best of his/her capacity. By being the gatekeeper for information, the medical professional-regardless if that means human or any future implementation of $\mathrm{AI}-$ acts as the guardian of the patient's autonomy. Moreover, it is crucial to take the belief system and expectations of the patient or his/her surrogate into account [42] to prevent a return to the paternalistic medicine of the past. That type of medicine was mostly based on calculations of non-maleficence and beneficence by physicians. Empirical studies, however, indicated that the trust of patients in the prognostication accuracy of physicians is rather low [43]. This finding is especially important when discussing irreversible decisions, such as withdrawing treatment after ranking quality of life higher than extending life at any cost. Patient-centered outcomes rarely are binary and can involve a broad range of expectations related to the self-perceived quality of life. Regarding AI models for prognostication, this fact requires more consideration for both the training samples for machine learning as well as for defining the types of output.

A difficult problem for shared decision-making is the discussion about probabilities while prognosticating future events. Regarding the uncertainty inherent in every prediction, probabilistic models are likely the best available instruments now. However, if one has to decide upon an individual case, i.e., sample size of 1 , probabilities are meaningless irrespective of the mathematical validity of the model [4]. Although human intuition may indicate otherwise [44], there is no objective difference between a predicted mortality of 10 and $90 \%$ for the assessment of an individual case. Thus, any dispute about how to act on probabilistic information for an individual, especially on when and how to invest resources, cannot be resolved with data from AI models alone. Instead, there has to be a mutual agreement between the patient or surrogate and the physician about the interpretation of probabilities. A disagreement has the potential of interfering with the principles of autonomy and justice. A solution to that dilemma is an observation period to obtain longitudinal data for the individual patient and, thereby, reduce the uncertainty of prognostication [1].

\section{The principle of explicability}

In addition to the four principles of medical ethics, recent guidelines for an ethical AI also dealt with the issue of explicability, i.e., transparency of models in producing outputs based on specific inputs [13,31]. The interplay and potential tensions between these five principles is depicted in Fig. 1. Of note, the absence of insight into the mechanisms of data processing by AI models is not fundamentally different from the opacity of human thinking [10]. Without critical reflection, algorithmic tools, such as conventional prognostication scores, are handled by intensivists like "black boxes" [45]. However, humans can be requested to reason and justify their conclusions if there is a lack of certainty or trust. In contrast, there has not been a design framework yet in place that creates AI systems supporting a similar relationship. Current research into illustrating the models' decision-making process in more transparent ways aims at distilling ANNs into graphs for interpretative purposes, such as decision trees [46], defining decision boundaries [47], approximating model predictions locally with interpretable models [48], or analyzing the specific impact of individual parameters on predictions [49]. Full explicability may not always be possible and other measures to audit outputs need to be implemented to assure that the principles of medical ethics are respected [31]. Trust in 


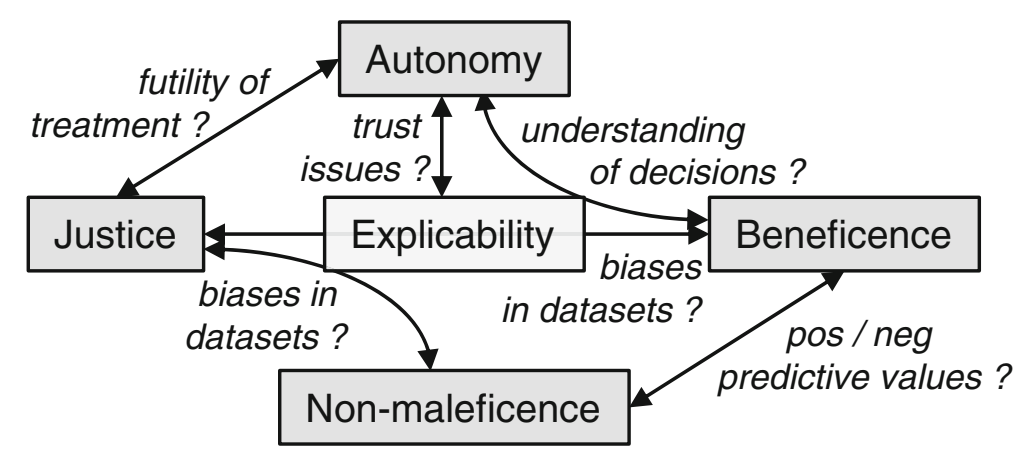

Fig. 1 The interplay and potential areas of tensions between the four principles of medical ethics as well as with the principle of explicability

the working of AI algorithms and the ability to interact with them would enhance the patients' confidence that is required for shared decision-making. Moreover, model transparency-as far as this might be achieved-also helps to clarify questions of moral and legal accountability in case of mistakes [10].

The design and deployment of AI systems also evoked discussions about societal values and fundamental rights with explicability being the focus of much controversy. Some scientists recommend sacrificing the power of AI models in favor of explicability to foster social trust and prevent domination by unaccountable models or algorithms [10]. Of note, if machine learning is considered empiricism, this issue has already been picked up by Aristotle more than two millennia ago. Large parts of the evidence base in medicine resulted from empirical studies. However, empiricism in prognostication that informs irreversible decisions in intensive care requires rules and boundaries. A quantitative assessment of uncertainty plays a major role in this regard $[19,50]$.

\section{Privacy and confidentiality}

Privacy and confidentiality are still important values to protect human dignity as a fundamental right in many countries. New guidelines for data processing, notably the general data protection regulation (GDPR) in Europe, also contain demands to communicate risks created by the processing of patients' data including profiling and its consequences [26].

\section{A pathway to an ethical Al-based prognostication}

The guidelines for a trustworthy AI by the European Commission [31] list several requirements to translate the above ethics principles into practice (Table 1). Some of

Table 1 Requirements for the implementation of ethics principles for a trustworthy Al (based on

\begin{tabular}{ll}
$[31])$ & \\
\hline Leading ethics principle & Requirement for implementation \\
\hline Beneficence & Data stewardship, accountability \\
Non-maleficence & Technical robustness \\
Justice & Fairness, societal wellbeing \\
Autonomy & Human agency and oversight \\
Explicability & Transparency \\
\hline
\end{tabular}


these requirements are directly derived from ethical principles, such as transparency and explicability. Others are prerequisites for implementing these principles, such as the requirement for human agency to support patients' autonomy.

\section{Technical issues}

Based on these guidelines, we suggest a checklist to assess AI systems for the purpose of prognostication in intensive care (Fig. 2). This set of questions consists of four topics-medical, technical, patient-centered, and system-centered. After defining the purpose for prognostication, e.g., change of medical management, the selection of a suitable AI model is crucial. This concerns input and output data types, the architecture of the AI model, such as ANN or random forest, as well as the dataset used for training. The origin and composition of this dataset must be clearly defined to identify explicit and implicit biases. Ideally, the training dataset is from the same cultural background to minimize distributional shifts. Since no gold standard for AI model design has yet been elaborated, the process of model development might involve several iterations trialing various models to optimize performance. Human expertise and creativity might still be required for this task. Maximum explicability of the model should be sought by quantifying the role of individual parameters [49] and decision boundaries analysis [47]. This approach is not unlike the training of intensivists-the trainees initially possess a set of basic skills which they refine according to performance measures. While doing this, they have to explain their decisions including their reasoning.

The benefit of AI-based prognostication models is determined by their accuracy which can be described by the calibration at the cohort level and the quantification of discriminatory power and predictive uncertainty [29]. To adhere to the ethical principle of non-maleficence, model predictions cannot be actionable without confidence values for assessing uncertainty and, thereby, risks [19]. There is no standardized approach to estimate predictive uncertainty that results from the model architecture and the machine learning algorithm itself. However, recent research indicate that, for example, training multiple ANNs using random initialization and ensembling predictions could be a useful method to obtain quantitative estimates for uncertainty [29].

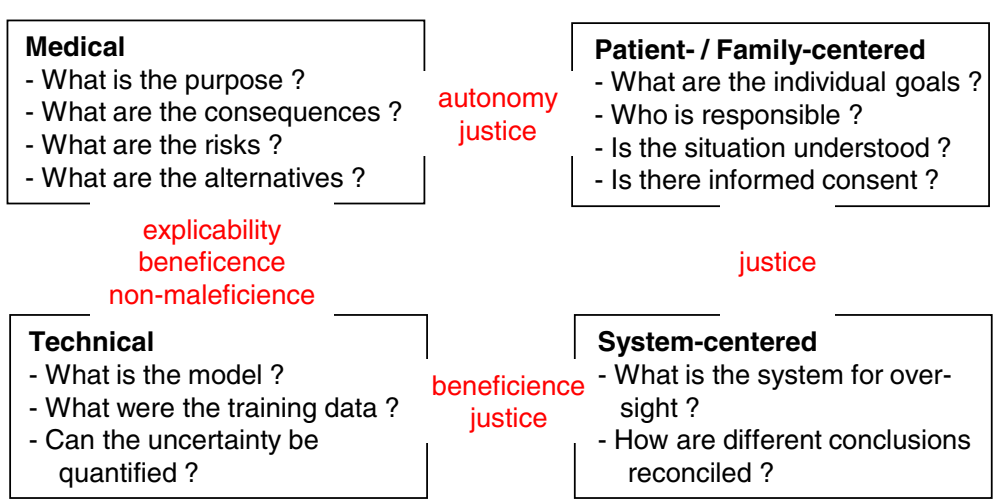

Fig. 2 Classes of questions for evaluating Al-based prognostication models (the main ethical principles related to specific topics are depicted in red) 
Issues of decision-making

We think that shared decision-making with patients or their surrogates is the best way to finally decide upon the consequences of information from AI-based prognostication models. This approach also guarantees human oversight and, thereby, assigns responsibilities to the physician and the patient or surrogate. Assuming that the AI model was formally appropriate and tested, differences in predictions between that model and those by the medical staff should be dealt with by seeking additional (human) opinion, e.g., in multidisciplinary team meetings. This also concerns conflicts between patients or surrogates and medical staff in interpreting results [41].

\section{Societal issues}

Governments have established systems for oversight to assure the competency of medical staff and, thereby, support trust in the health care system. New AI technologies should face a similar regulatory scrutiny that encompasses the design, configuration and operation of algorithms [26]. Moreover, medical staff should be trained to evaluate and supervise these systems as well as to discuss the characteristics of AI, including potential errors, with patients and surrogates. Finally, the general public should be educated in dealing with AI to better understand the merits and dangers of AI-based prognostication and become capable of joining the decision-making process when needed.

Table 2 summarizes the main steps of implementing AI models for prognostication as well as potential mistakes.

\section{Discussion and conclusions}

Data-driven AI is considered a disruptive and transformative technology in medicine. It provides the opportunity to process a multitude of patient-level data to detect and specify disease patterns. Although powerful tools are being developed with this technology to predict event rates and risks at the population level, the prognostication of future events, disease trajectories, or functional outcome for the individual patient remains a fundamental problem. An important reason is the probabilistic nature of all prediction models derived from cohort data which are then applied for the individual case. Moreover, the stochastic nature of the interplay between the patient's conditions and the environment adds a substantial amount of predictive uncertainty. Thus, individual prognostication is inherently uncertain. New information technologies can not eliminate but only reduce this

Table 2 Dos and don'ts while implementing Al-based prognostication models

\begin{tabular}{ll}
\hline Do & Don't \\
\hline Define individual goals & Rely on a single model \\
Clarify responsibilities & Use models without testing \\
Choose a suitable model & Make decisions in paternalistic ways \\
Assure technical robustness & Be cavalier with human oversight \\
Seek maximum transparency & Ignore data privacy issues \\
Quantify uncertainty & Underestimate the role of empiricism \\
Share decision-making & \\
Monitor performance and update model & \\
Contribute to research and education & \\
\hline
\end{tabular}


uncertainty. For the sake of prognostication in the individual, it is less important to set an "acceptable" threshold for the accuracy of a particular model at the population level, but to create a framework to describe and handle uncertainty.

In anticipation of imperfect predictions for the individual and, consequently, potentially wrong decisions, rules are required to protect the dignity of patients as well as the integrity of medical professionals. These rules are ingrained in laws and guidelines as well as ethical standards with the latter being regarded superior to the others. The ability to make decisions under conditions of uncertainty and in compliance with the rules of medical ethics is considered a crucial competency of intensivists. AI-based techniques which, one day, might develop from assist systems into "artificial intensivists" will need to acquire the same competency. Whereas the accuracy of prognostication and, eventually, decision-making are evaluated in the setup of clinical trials, ethical problems have not yet received sufficient attention when discussing AI.

The main principles of medical ethics are beneficence, non-maleficence, justice, and respect for patient's autonomy. Whereas compliance to beneficence and non-maleficence by AI models can be evaluated-at least partially-by standardized measures, such as sensitivity and specificity, compliance to the other two principles is more difficult to assess. Moreover, the latter two principles have the potential to be in conflict with each other and to turn individual interests against societal interests [32]. Importantly, the less well-understood mathematical characteristics of new AI technologies lead to a lack of transparency and problems with uncertainty quantification in the decision-making process. Both characteristics, however, are prerequisites to assess justice and implement the principle of patients' autonomy through knowledge and understanding. Of note, a fully autonomous decision-making system could serve justice best at the level of society. However, such an approach would violate the principle of human autonomy and be ignorant of empathy that is considered a fundamentally human trait. These problems (cf. Fig. 1) are not restricted to medical applications and requires solutions in a wider context [51].

The principle of patient's autonomy is widely seen as a major rule that governs the patient-physician relationship. It requires a comprehensive understanding of the medical issues to be dealt with by both sides. This is not realistic, especially when involving new technologies which are at the edge of today's knowledge. Thus, the principle of patient's autonomy has to be discussed in the context of trust in the competencies of medical professionals as well as in the system of oversight for new technologies. The concept of shared decision-making and the systems of legal responsibilities are based on this trust.

Currently, there are a large number of new guidelines on AI being issued by both professional and governmental institutions. These need to be synchronized and unified under universally accepted rules and limitations. This process will be iterative and consultative to take new developments into account. Moreover, educating the public as well as the members of the professions not traditionally involved in AI would create the foundation for a widespread acceptance of AI.

The implementation of new predictive AI technologies into the provision of intensive care medicine requires strict data governance measures which include safeguards for the integrity and quality of data. The governance of algorithms is also important. Many established prognostication methods can be considered "black boxes" when applied without in-depth knowledge. Thus, the status quo of prognostication cannot be 
regarded as a gold standard to evaluate new technologies. To guide a formal assessment of new prediction models, the performance of these models should be at least as good as that of experts or already established models as documented in clinical trials. This would allow for dynamic adjustments and ensure the quality of care at the systems level.

The current lack of explicability of many AI techniques is going to restrict their use to adjunct, e.g., decision-support, systems for a while. After conclusive evidence for their overall effectiveness and beneficence will become available, these new techniques will most likely turn into perceived standards. They will gain professional acceptance [52] and diverting from them will require justification [53]. Importantly, values in society evolve over time. Thus, continuous monitoring of AI model performance and patients' outcome should become mandatory to calibrate these measures against ethical standards.

In conclusion, new AI and machine learning techniques have the potential to improve prognostication in intensive care. However, they require further refinement before they can be introduced into daily practice. This encompasses technical problems, such as uncertainty quantification, inclusion of more patient-centered outcome measures and important ethical issues notably regarding hidden biases as well as the transparency of data processing and the explainability of results. Thereafter, AI models may become a valuable component of the intensive care team.

Abbreviations

Al: Artificial intelligence; AUROC: Area under the receiver operating curve

Acknowledgements

Not applicable

Authors' contributions

All authors contributed to collecting and discussing ideas and writing the manuscript. All authors read and approved the final manuscript.

Funding

Institutional.

Availability of data and materials

Not applicable.

Ethics approval and consent to participate

Not applicable.

Consent for publication

Not applicable.

Competing interests

The authors declare that they have no competing interests.

Author details

${ }^{1}$ Institute of Health Sciences at PTHV, Pallottistr. 3, 56179 Vallendar, Germany. ${ }^{2}$ Institute of Ethics at PTHV, Pallottistr. 3, 56179 Vallendar, Germany. ${ }^{3}$ Melbourne, Australia. ${ }^{4}$ Hadassah - Hebrew University Medical Center, POB 12000, 9112001

Jerusalem, Israel.

Received: 18 October 2019 Accepted: 28 November 2019

Published online: 10 December 2019

References

1. Kon AA, Shepard EK, Sederstrom NO, Swoboda SM, Marshall MF, Birriel B, Rincon F (2016) Defining futile and potentially inappropriate interventions: a policy statement from the Society of Critical Care Medicine Ethics Committee. Crit Care Med 44:1769-1774

2. Anesi GL, Admon AJ, Halpern SD, Kerlin MP (2019) Understanding irresponsible use of intensive care unit resources in the USA. Lancet Respir Med 7:605-612 
3. Castela Forte J, Perner A, van der Horst ICC (2019) The use of clustering algorithms in critical care research to unravel patient heterogeneity. Intensive Care Med 45:1025-1028

4. Kent DM, Steyerberg E, van Klaveren D (2018) Personalized evidence based medicine: predictive approaches to heterogeneous treatment effects. BMJ 363:k4245

5. Meiring C, Dixit A, Harris S, MacCallum NS, Brealey DA, Watkinson PJ, Jones A, Ashworth S, Beale R, Brett SJ, Singer M, Ercole A (2018) Optimal intensive care outcome prediction over time using machine learning. PLoS One 13:e0206862

6. Hinton G (2018) Deep learning - a technology with the potential to transform health care. JAMA 320:1101-1102

7. McWilliams CJ, Lawson DJ, Santos-Rodriguez R, Gilchrist ID, Champneys A, Gould TH, Thomas MJ, Bourdeaux CP (2019) Towards a decision support tool for intensive care discharge: machine learning algorithm development using electronic healthcare data from MIMIC-III and Bristol, UK. BMJ Open 9:e025925

8. Nanayakkara S, Fogarty S, Tremeer M, Ross K, Richards B, Bergmeir C, Xu S, Stub D, Smith K, Tacey M, Liew D, Pilcher D, Kaye DM (2018) Characterising risk of in-hospital mortality following cardiac arrest using machine learning. PLoS Med 15:e1002709

9. Pirracchio R, Petersen ML, Carone M, Rigon MR, Chevret S, van der Laan MJ (2015) Mortality prediction in intensive care units with the Super ICU Learner Algorithm (SICULA): a population-based study. Lancet Respir Med 3:42-52

10. London AJ (2019) Artificial intelligence and black-box medical decisions: accuracy versus explainability. Hastings Cent Rep 49:15-21

11. Jaderberg M, Czarnecki WM, Dunning I, Marris L, Lever G, Castañeda AG, Beattie C, Rabinowitz NC, Morcos AS, Ruderman A, Sonnerat N, Green T, Deason L, Leibo JZ, Silver D, Hassabis D, Kavukcuoglu K, Graepel T (2019) Humanlevel performance in 3D multiplayer games with population-based reinforcement learning. Science 364:859-865

12. Ting DSW, Pasquale LR, Peng L, Campbell JP, Lee AY, Raman R, Tan GSW, Schmetterer L, Keane PA, Wong TY (2019) Artificial intelligence and deep learning in ophthalmology. Br J Ophthalmol 103:167-175

13. Academy of Medical Royal Colleges (2019) Artificial Intelligence in healthcare.

14. Komorowski M (2019) Artificial intelligence in intensive care: are we there yet? Intensive Care Med. 45:1298-1300

15. LeCun Y, Bengio Y, Hinton G (2015) Deep learning. Nature 521:436-444

16. Ghahramani Z (2015) Probabilistic machine learning and artificial intelligence. Nature 521:452-459

17. Hochreiter S, Schmidhuber J (1997) Long short-term memory. Neural Comput 9:1735-1780

18. Steinruecken C, Smith E, Janz D, Lloyd J, Ghahramani Z (2019) The automatic statistician. In: Kotthoff L, Vanschoren J (eds) Hutter F. Springer, Automated Machine Learning

19. Begoli E, Bhattacharya T, Kusnezov D (2019) The need for uncertainty quantification in machine-assisted medical decision making. Nat Machine Intell 1:20-23

20. Rajkomar A, Oren E, Chen K, Dai AM, Hajaj N, Hardt M, Liu PJ, Liu X, Marcus J, Sun M, Sundberg P, Yee H, Zhang K, Zhang Y, Flores G, Duggan GE, Irvine J, Le Q, Litsch K, Mossin A, Tansuwan J, Wang D, Wexler J, Wilson J, Ludwig D, Volchenboum SL, Chou K, Pearson M, Madabushi S, Shah NH, Butte AJ, Howell MD, Cui C, Corrado GS, Dean J (2018) Scalable and accurate deep learning with electronic health records. npj Digital Med 1:18

21. Cahan EM, Hernandez-Boussard T, Thadaney-Israni S, Rubin DL (2019) Putting the data before the algorithm in big data addressing personalized healthcare. NPJ Digit Med 2:78

22. Andersen FH, Flaatten H, Klepstad P, Romild U, Kvale R (2015) Long-term survival and quality of life after intensive care for patients 80 years of age or older. Ann Intensive Care 5:53

23. Vest MT, Murphy TE, Araujo KL, Pisani MA (2011) Disability in activities of daily living, depression, and quality of life among older medical ICU survivors. Health Qual Life Outcomes 9:9

24. Vermeulen J, Neyens JC, van Rossum E, Spreeuwenberg MD, de Witte LP (2011) Predicting ADL disability in communitydwelling elderly people using physical frailty indicators. BMC Geriatr 11:33

25. Lawson RA, Yarnall AJ, Duncan GW, Breen DP, Khoo TK, Williams-Gray CH, Barker RA, Collerton D, Taylor JP, Burn DJ, ICICLE-PD study group (2016) Cognitive decline and quality of life in incident Parkinson's disease. Parkinsonism Relat Disord 27:47-53

26. Mittelstadt BD, Allo P, Taddeo M, Wachter S, Floridi L (2016) The ethics of algorithms: mapping the debate. Big Data \& Society 2:1-21

27. Gomez E (2018) Assessing the impact of machine intelligence on human behaviour. Proceedings of 1st HUMAINT workshop, Barcelona, Spain, March 5-6, 2018. Luxembourg: Publications Office of the European Union.

28. Finlayson SG, Chung HW, Kohane IS, Beam AL (2019) Adversarial attacks against medical deep learning systems. arXiv: $1804.05296 \mathrm{v} 3$

29. Ovadia Y, Fertig E, Ren J, Nado Z, Sculley D, Nowozon S, Dillon JV, Lakshminarayanan B, Snoek J (2019) Can you trust your model's uncertainty? Evaluating predictive uncertainty under dataset shift. arXiv:1906.02530v1

30. Winfield AF, Michael K, Pitt J, Evers V (2019) Machine ethics: the design and governance of ethical Al and autonomous systems. Proc IEEE 107:509-517

31. High-Level Expert Group on Artificial Intelligence (2019) Ethics guidelines for trustworthy Al. European Commission, Brussels

32. Gillon R (2015) Defending the four principles approach as a good basis for good medical practice and therefore for good medical ethics. J Med Ethics 41:111-116

33. Hwang DY, White DB (2018) Prognostication and ethics. In: Shutter L, Molyneaux BJ (eds) Neurocritical Care. Oxford University Press

34. Reddy BK, Delen D (2018) Predicting hospital readmission for lupus patients: an RNN-LSTM-based deep-learning methodology. Comput Biol Med. 101:199-209

35. Dumas F, Bougouin W, Cariou A (2019) Cardiac arrest: prediction models in the early phase of hospitalization. Curr Opin Crit Care 25:204-210

36. Le Gall JR, Neumann A, Hemery F, Bleriot JP, Fulgencio JP, Garrigues B, Gouzes C, Lepage E, Moine P, Villers D (2005) Mortality prediction using SAPS II: an update for French intensive care units. Crit Care. 9:R645-R652

37. Seymour CW, Kennedy JN, Wang S, Chang CH, Elliott CF, Xu Z, Berry S, Clermont G, Cooper G, Gomez H, Huang DT, Kellum JA, Mi Q, Opal SM, Talisa V, van der Poll T, Visweswaran S, Vodovotz Y, Weiss JC, Yealy DM, Yende S, Angus DC (2019) Derivation, validation, and potential treatment implications of novel clinical phenotypes for sepsis. JAMA. 2019 May 19. 
38. Liu R, Greenstein JL, Granite SJ, Fackler JC, Bembea MM, Sarma SV, Winslow RL (2019) Data-driven discovery of a novel sepsis pre-shock state predicts impending septic shock in the ICU. Sci Rep 9:6145

39. Beauchamps TL, Childress JF (1994) Principles of biomedical ethics. Med Clin North Amer 80:225-243

40. Bailey J, Burch M (2013) Ethics for behavior analysts, 2nd edn. Routledge, New York

41. Bosslet GT, Pope TM, Rubenfeld GD, Lo B, Truog RD, Rushton CH, Curtis JR, Ford DW, Osborne M, Misak C, Au DH, Azoulay E, Brody B, Fahy BG, Hall JB, Kesecioglu J, Kon AA, Lindell KO, White DB (2015) An official ATS/AACN/ACCP/ ESICM/SCCM policy statement: responding to requests for potentially inappropriate treatments in intensive care units. Am J Respir Crit Care Med 191:1318-1330

42. Scheunemann LP, Ernecoff NC, Buddadhumaruk P, Carson SS, Hough CL, Curtis JR, Anderson WG, Steingrub J, Lo B, Matthay M, Arnold RM, White DB (2019) Clinician-family communication about patients' values and preferences in intensive care units. JAMA Intern Med.179(5):676-684.

43. Zier LS, Burack JH, Micco G, Chipman AK, Frank JA, White DB (2009) Surrogate decision makers' responses to physicians' predictions of medical futility. Chest 136:110-117

44. Joynt GM, Lipman J, Hartog C, Guidet B, Paruk F, Feldman C, Kissoon N, Sprung CL (2015) The Durban World Congress Ethics Round Table IV: health care professional end-of-life decision making. J Crit Care 30:224-230

45. Cannesson M, Shafer SL (2016) All boxes are black. Anesth Analg. 122:309-317

46. Frosst $N$, Hinton $G$ (2017) Distilling a neural network into a soft decision tree. arXiv:1711.09784

47. Li Y, Richtarik P, Ding L, Gao X (2018) On the decision boundary of deep neural networks. arXiv:1808.05385

48. Zhang Z, Beck MW, Winkler DA, Huang B, Sibanda W, Goyal H (2018) Opening the black box of neural networks: methods for interpreting neural network models in clinical applications. Ann Transl Med. 6:216

49. Lundberg SM, Lee SI (2017) A unified approach to interpreting model predictions. Proceedings of the Conference on Neural Information Processing Systems (NIPS 2017), Long Beach, CA, USA

50. Raghu M, Blumer K, Sayres R, Obermeyer Z, Kleinberg R, Mullainathan S, Kleinberg J (2019) Direct uncertainty prediction for medical second opinions. Proceedings of the 36th International Conference on Machine Learning, Long Beach, CA, USA.

51. Whittlestone J, Alexandrova A, Nyrup, R, Cave, S (2019) The role and limits of principles in Al ethics. Proceedings 2019 AAAl/ACM Conference on Al, Ethics, and Society.

52. UK Government (2019) Code of conduct for data-driven health and care technology. https:/www.gov.uk/government/ publications/code-of-conduct-for-data-driven-health-and-care-technology/initial-code-of-conduct-for-data-driven-healthand-care-technology. Accessed 14 Aug 2019.

53. Biller-Andorno N, Biller A (2019) Algorithm-aided prediction of patient preferences - an ethics sneak peek. N Engl J Med. 381:1480-1485

\section{Publisher's Note}

Springer Nature remains neutral with regard to jurisdictional claims in published maps and institutional affiliations.

\section{Submit your manuscript to a SpringerOpen ${ }^{\circ}$ journal and benefit from:}

- Convenient online submission

- Rigorous peer review

- Open access: articles freely available online

High visibility within the field

- Retaining the copyright to your article

Submit your next manuscript at $\boldsymbol{\nabla}$ springeropen.com 